\title{
Preparation and Property of PVDF Ultrafiltration Membrane Modified by Graphene
}

\author{
Beifu WANG ${ }^{1, a}$, Qingtao WU ${ }^{1, b}$ \\ ${ }^{1}$ Haida Southroad No. 1, Changzhi Island, Lincheng District, Zhoushan City, Zhejiang \\ province,China \\ awangbeifu@126.com, ${ }^{b}$ wqt137@126.com
}

Keywords: graphene; ultrafiltration membrane; separation performance; hydrophilic; tensile strength

Abstract. In order to improve the hydrophilicity and mechanical strength of the membrane, the modified PVDF ultrafiltration membranes were prepared by the phase inversion method with different contents of nano graphene dispersed uniformly in the PVDF casting solution. In the experiment, the effect of graphene on the separation performance, contact angle, zeta potential and tensile strength of the membranes were investigated, and the flux recovery rate and flux decline rate were also investigated. The crystal structure of the membranes were characterized by $\mathrm{X}$ ray diffraction (XRD) and thermal analysis (DSC). The results showed that the performance of pore size, porosity and pure water flux of the modified membranes were improved. The tensile strength, hydrophilic and anti-fouling properties of the membranes were also improved. The separation performance, hydrophilic and anti-fouling properties of the modified membranes were the best when the addition content of graphene was $0.5 \%$. Graphene had no effect on the crystallinity of the membranes, and XRD found that the graphene in the casting solution was physical blend.

\section{Introduction}

The surface energy of the hydrophobic polymer PVDF was low. PVDF membrane was easy to be polluted in the process of sewage treatment, seriously reduced the membrane life and increased the operation cost. To modify the membrane material was the fundamental way to improve the fouling phenomenon of membranes in recent years. Inorganic nanoparticles were mixed with polyvinylidene fluoride to prepare the organic-inorganic hybrid membranes, which was a hot issue for researchers[1,2]. As a new type of carbon nano material, graphene had a high specific surface area and excellent mechanical property[3,4]. The researchers had prepared a great deal of composite materials with excellent properties. Zhang had prepared PET-Gr composite by dispersed graphene in the PET matrix through the melt blending method[5]. Ma and other firstly inserted the polymer silicon resin into the functionalized graphene oxide nano sheet (FGONs) and prepared the FGONs / silicon resin composite using the solvent effect by solution mixing method[6]. Under this situation, the graphene modified PVDF ultrafiltration membranes were prepared through phase inverse method.

Materials and Methods. The graphene modified PVDF modified membranes were prepared through the phase inverse method. The characterization of performance of the membranes are as follows:

Separation Performance. The index of separation performance of the membranes were mainly pure water flux, rejection, porosity and pore size.

Hydrophilic Property. The index of hydrophilic property of the membranes were contact angle and surface zeta potential.

Mechanical Property. The mechanical property of membranes was expressed by tensile strength. Anti-fouling Property. The index of anti-fouling property were flux decline rate and flux recovery rate.

Crystal Structure. The crystal structure of the membranes were characterized by $\mathrm{X}$ ray diffraction (XRD) and thermal analysis (DSC). 


\section{Results and Discussion.}

\section{Separation Performance.}

Table 1 Separation performance of membranes

\begin{tabular}{ccccc}
\hline Sample & Porosity[\%] & Pore radius $\left[\times 10^{-8} \mathrm{~m}\right]$ & Water flux $\left[\mathrm{L} \cdot \mathrm{h}^{-1} \cdot \mathrm{m}^{-2}\right]$ & Rejection[\%] \\
\hline PVDF & 66 & 8.00 & 484 & 96 \\
PVDF $_{1}$ & 66 & 8.82 & 701 & 97 \\
PVDF $_{3}$ & 67 & 9.00 & 727 & 97 \\
PVDF $_{5}$ & 70 & 8.89 & 835 & 97 \\
PVDF $_{7}$ & 67 & 8.34 & 654 & 96 \\
$\mathrm{PVDF}_{10}$ & 68 & 8.52 & 636 & 96 \\
\hline
\end{tabular}

Note: PVDF was the unmodified membrane, $\mathrm{PVDF}_{1}$ was the modified membrane with $0.1 \%$ graphene and as above.

From the Table 1, it can be known that the separation performance of the graphene modified membranes was improved compared with the unmodified PVDF film. The porosity and pore size were also increased. The pure water flux was increased from $584 \mathrm{~L} \cdot \mathrm{h}^{-1} \cdot \mathrm{m}^{-2}$ to $835 \mathrm{~L} \cdot \mathrm{h}^{-1} \cdot \mathrm{m}^{-2}$, the retention rate about bovine serum albumin had no significant change.

\section{Hydrophilic Property.}

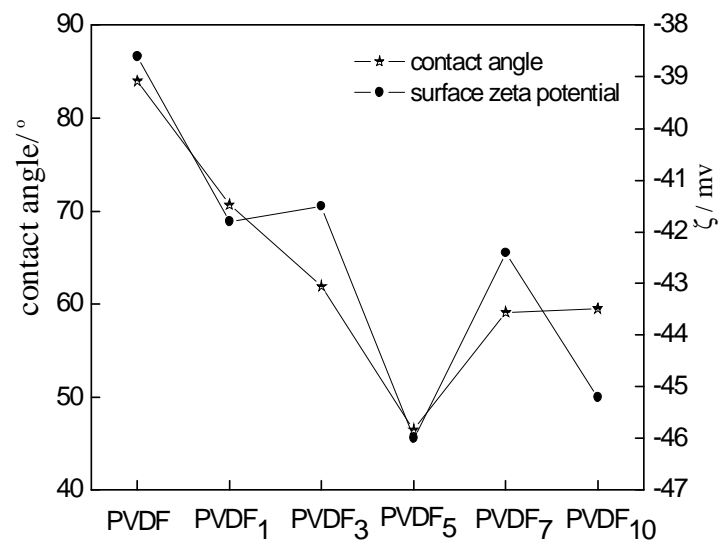

Fig. 1 Contact angle and surface zeta potential of membranes

From the Fig. 1, it can be known that the contact angle of the modified membranes were decreased and the absolute value of the surface zeta potential of the modified membranes was increased. Therefore, the graphene had significant effect on the hydrophilicity of the membranes.

\section{Mechanical Properties.}

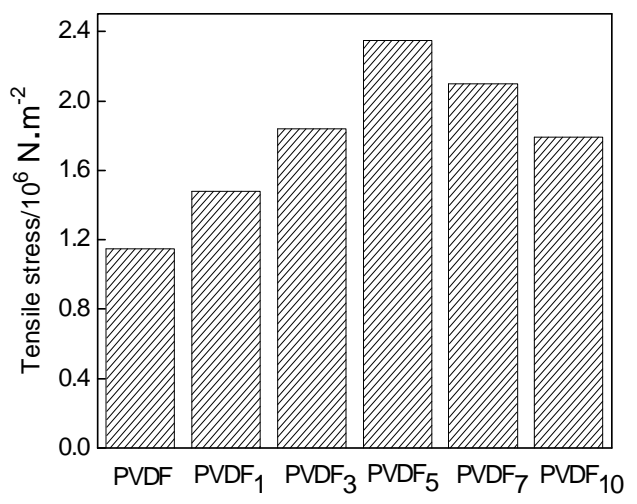

Fig. 2 The mechanical property of membranes 
As we can see from Fig. 2, graphene could improve the mechanical property of the membranes, the tensile strength of the films was increased with the amount of graphene.

\section{Anti-fouling Property.}

Table 2 Flux recovery rate of membranes

\begin{tabular}{cccc}
\hline Sample & $\mathrm{J}_{0}\left[\mathrm{~L} \cdot \mathrm{h}^{-1} \cdot \mathrm{m}^{-2}\right]$ & $\mathrm{J}_{\mathrm{w}}\left[\mathrm{L} \cdot \mathrm{h}^{-1} \cdot \mathrm{m}^{-2}\right]$ & FRR[\%] \\
\hline PVDF & 484 & 213 & 44 \\
PVDF $_{1}$ & 701 & 421 & 60 \\
PVDF $_{3}$ & 727 & 451 & 62 \\
PVDF $_{5}$ & 835 & 635 & 76 \\
PVDF $_{7}$ & 654 & 477 & 73 \\
PVDF $_{10}$ & 636 & 458 & 72 \\
\hline
\end{tabular}

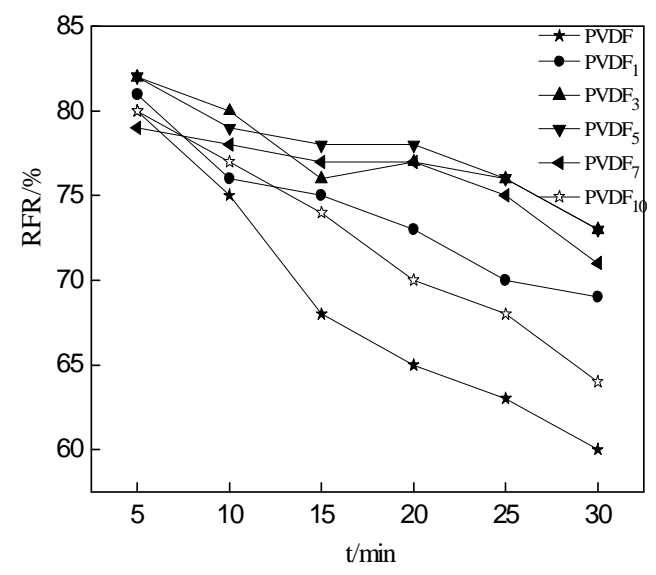

Fig. 3 The rate of flux decline of membranes

As was shown in Table 2 and Fig.3, the flux recovery rate of the modified membranes was improved, and the flux decline rate was weaken. Thus, the anti-fouling property was improved by graphene.

Crystal Structure.

Table 3 Melting temperature, heat of fusion and crystallinity of membranes

\begin{tabular}{cccc}
\hline Sample & $\mathrm{T}_{\mathrm{m}}\left[{ }^{\circ} \mathrm{C}\right]$ & $\Delta \mathrm{H}_{\mathrm{m}}\left[\mathrm{J} \cdot \mathrm{g}^{-1}\right]$ & $\mathrm{X}[\%]$ \\
\hline $\mathrm{PVDF}$ & 163.3 & 44.83 & 42.69 \\
$\mathrm{PVDF}_{1}$ & 163.7 & 42.63 & 40.60 \\
$\mathrm{PVDF}_{3}$ & 164.1 & 44.42 & 42.30 \\
$\mathrm{PVDF}_{5}$ & 164.9 & 45.55 & 43.38 \\
$\mathrm{PVDF}_{7}$ & 166.2 & 42.66 & 40.63 \\
$\mathrm{PVDF}_{10}$ & 163.8 & 42.93 & 40.89 \\
\hline
\end{tabular}




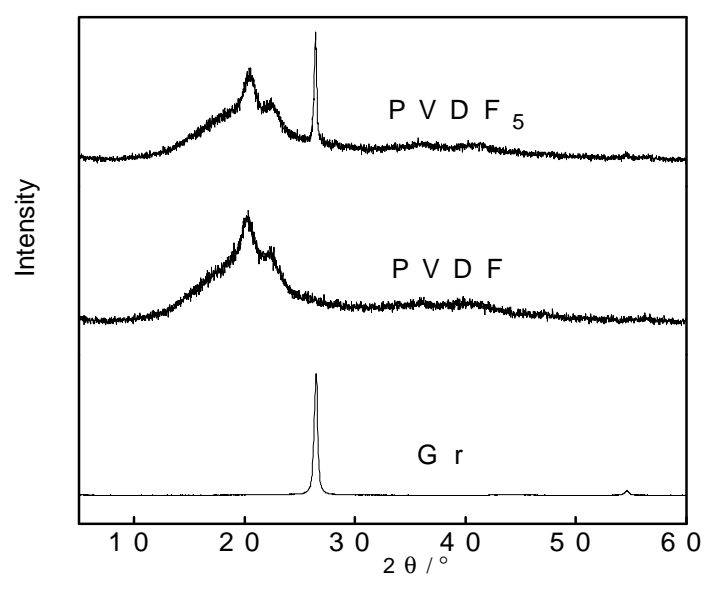

Fig. 4 X-ray diffractograms of membranes

From Table 3, it can be known that graphene had little effect on the crystallinity of the UF membrane. In the Fig. 4, a strong peak at 26 degree that was consistent with the peak position of the graphene powder and graphene was added into the casting solution as a physical blending method.

\section{Summary}

The graphene modified membranes possessed a better separation performance, mechanical property, hydrophilicity and anti-fouling property. The result showed that the optimum addition content of the graphene was $0.5 \%$. Graphene had little effect on the crystallinity of the membranes and was added into the casting solution as a physical blending method.

\section{References}

[1] Law Yong Ng, Abdul Wahab Mohammad, Choe Peng Leo, et al. Desalination Vol.308(2013), p.15-33

[2] Bashiru Kayode Sodipo, Azlan Abdul Aziz. Ultrasonics Sonochemistry Vol.23(2015), p.354-359

[3] Novoselov K, Geim A, Morozov S, et al. Science Vol.306(2004), p.666-669

[4] Meyer J C, Geim A K, Katsnelson M I, et al. Nature Vol.446(2007), p.446: 63-64

[5] Zhang H B, Zheng W G, Yan Q, et al. Polymer Vol.51(2010), p.1191-1196

[6] Ma W S, Li J, Zhao X S. Journal of Materials Science Vol.48(2013), p.5287-5294 